\title{
Identification and characterization of gastrointestinal hormone immunoreactive cells in the skin and parotoids of Chinese toad Bufo gargarizans
}

\author{
Huan Wang, Yuan-Yuan Wu, Rui Zhang, Xue Zhu, Sheng-Zhou Zhang \\ Anhui Key Laboratory of Biotic Environment and Ecological Safety, College of Life Sciences, \\ Anhui Normal University, Wuhu, China
}

\begin{abstract}
The skin and skin secretion of Chinese toad Bufo gargarizans have long been used in traditional Chinese medicine. However, the exact types and location of bioactive substances in Bufo gargarizans skin still have not been fully elucidated. The aim of the study was to investigate the distribution and density of six types of gastrointestinal (GI) hormone immunoreactive (IR) cells in the skin and parotoids of Bufo gargarizans. Immunohistochemistry was used for qualitative and semiquantitative analysis of GI hormone presence in the dorsal and ventral skin, and parotoids of eight adult Chinese toads. Six types of IR cells were found: serotonin (5-HT), glucagon (GLU), gastrin (GAS), somatostatin (SS), pancreatic polypeptide (PP) and neuropeptide Y (NPY) IR cells. They were mainly present in the epidermis and skin glands. 5-HT-IR cells were distributed in all layers of epidermis and glands, with higher density in the glands. Glucagon was prominently expressed in the epidermis and the bottle-shaped glands of parotoids; however, it was not present in the granular glands of skin and parotoids. The distributions of GAS and SS-IR cells were similar since they were present mainly in mucous, granular and bottle-shaped glands, while these cell types were absent in the differentiated glands of parotoids. PP-IR cells were predominant in the granular glands and the bottle-shaped glands. The expression of NPY was high in epidermal stratum granulosum and mucous glands of the dorsal skin, the bottle-shaped glands and differentiated glands of parotoids, while NPY-IR was rarely seen in the granular glands of ventral skin, and not present in the granular glands of dorsal skin and parotoids. The expression of several types of GI hormones in the skin and parotoids of Bufo gargarizans varies depending on tissue and type of glands. (Folia Histochemica et Cytobiologica 2014, Vol. 52, No. 2, 149-156)
\end{abstract}

Key words: Bufo gargarizans; Chinese toad; skin; epidermis; parotoids; gastrointestinal hormones; immunohistochemistry

\section{Introduction}

Amphibian skin and its secretion contain a wide variety of bioactive substances with various functions, which are the guarantee of amphibian's survival, and they are also a large drug storehouse yet to be developed [1]. Interestingly, numerous biogenic amines

Correspondence address: S.Z. Zhang

College of Life Sciences,

Anhui Normal University,

1 Beijing East Road,

Wuhu, Anhui Province 241000,

People's Republic of China;

e-mail: szzhang@mail.ahnu.edu.cn and biologically active peptides primarily found in the brain and gastrointestinal (GI) tract are also distributed in amphibian skins. Kramer detected serotonin (5-HT, 5-hydroxytryptamine) in the skin poison glands of three batrachian by histochemical method [2]. Later on, some scholars have also demonstrated the presence of 5-HT in the skin of Rana ridibunda and Bombina orientalis by immunohistochemistry and fluorescent histochemistry [3, 4]. de Magistris et al. reported the existence of gastrin (GAS), pancreatic polypeptide (PP) and Vasoactive Intestinal Peptide (VIP) positive substances in the APUD system of amphibian skin by the use of a sequence-specific radioimmunoassay and gel-chromatography [5]. Liu et al. identified the cholecystokinin (CCK) in the skin secretions of frog 
Table 1. Source and dilutions of the gastrointestinal hormone antisera

\begin{tabular}{|c|c|c|c|}
\hline Hormone antisera & Code No. & Dilution & Source \\
\hline Rabbit anti-serotonin (5-HT) & ZA-0231 & $1: 100$ & ZSGB Corp. \\
\hline Rabbit anti-glucagon (GLU) & ZA-0119 & 1:100 & Same as above \\
\hline Rabbit anti-gastrin (GAS) & ZA-0115 & $1: 75$ & Same as above \\
\hline Rabbit anti-somatostatin (SS) & ZA-0232 & 1:100 & Same as above \\
\hline Rabbit anti-pancreatic polypeptide (PP) & ZA-0211 & 1:100 & Same as above \\
\hline Rabbit anti-neuropeptide Y (NPY) & BA0355 & $1: 100$ & Boster Corp. \\
\hline
\end{tabular}

ZSGB Corp., Beijing, China; Boster Corp., Wuhan, China

Rana nigrovittata by the use of cDNA cloning method [6]. Li et al. detected the immunoreactive (IR) for 5-HT, GAS, PP, somatostatin (SS) and pancreatic glucagon (GLU) in the skin of Rana rugulosa by immunohistochemical (IHC) method [7].

Chinese toad Bufo gargarizans belongs to the bufonidae, anuran amphibians, which is widely distributed in China. The processed products Chan $\mathrm{Pi}$ (the toad skin) and Chansu (arenobufagin) which are the extracts from the dried toad skin and the white whey of parotoids are used to treat cardiovascular and cerebrovascular diseases and certain types of cancer, and proved to have antipyretic, detoxicant, diuretic, stasis-eliminative, and pus-discharging properties [8]. Chansu is also one of the major components of Kyushin, a traditional Chinese medicine [9]. Cinobufacini (Huachansu), a Chinese medicine prepared from Chan $\mathrm{Pi}$, has been widely used in clinical therapy for various cancers in China [10].

Several classes of compounds were isolated from the skin of Bufo gargarizans, including peptides, steroids, indole alkaloids, bufo gargarizanines, organic acids, and others [8]. However, there are no data about the exact types and location of bioactive peptides in Bufo gargarizans skin. We reported here for the first time that six types of important GI hormones were detected in the skin and parotoids of Bufo gargarizans by IHC technique. The presented data can deepen the understanding of the physiological function and biochemical composition of the skin and parotoids, and provide a reference for the development and utilization of Chansu and Chan Pi.

\section{Material and methods}

Specimens and tissue sections. Eight adult Chinese toads Bufo gargarizans weighing 150-200 $\mathrm{g}$ were collected in the suburb of Wuhu, Anhui province of China. They were sacrificed with phlebotomy from head and small pieces of samples (approximately $0.5 \mathrm{~cm}^{2}$ ) were taken from the dorsal skin, ventral skin and parotoids. The samples were cleaned with $0.70 \%$ saline, fixed in Bouin's fluid for $48 \mathrm{~h}$, and then dehydrated through graded ethanol series and embedded in paraffin. Finally, 5-6 $\mu \mathrm{m}$ thick sections were obtained and processed for IHC staining.

Antisera and reagents. The details of six types of antisera are listed in Table 1. Streptavidin-Biotin-Peroxidase Complex (SABC) IHC kit was purchased from Boster Corp. (Wuhan, China).

Immunohistochemical staining. Each representative section was deparaffinized, rehydrated and immunostained with conventional methods. Endogenous peroxidase activity was blocked by incubating the sections with $3 \% \mathrm{H}_{2} \mathrm{O}_{2}$ for $20 \mathrm{~min}$, washed in distilled water and then treated with phosphate -buffered saline (PBS, $10 \mathrm{mM}, \mathrm{pH}$ 7.4) for $5 \mathrm{~min}$. Blocking of the non-specific reaction was performed with normal goat serum prior to overnight incubation at $4^{\circ} \mathrm{C}$ with the primary antiserum (Table 1). After rinsing in PBS $(3 \times 3 \mathrm{~min})$, the sections were incubated at room temperature with biotinylated goat anti-rabbit IgG secondary antibody for $2 \mathrm{~h}$. After washing with PBS and subsequent incubation for $2 \mathrm{~h}$, the Streptavidin-Biotin-Peroxidase Complex was applied. After washes, the peroxidase reaction was carried out in DAB solution containing $0.01 \%$ of $\mathrm{H}_{2} \mathrm{O}_{2}$ in Tris- $\mathrm{HCl}$ buffer $(50 \mathrm{mM}$, $\mathrm{pH}$ 7.6) and subsequently washed with distilled water. After light counterstaining with Mayer's hematoxylin, the sections were dehydrated and cover-slipped. Negative control was carried out by incubating sections with PBS instead of the primary antiserum. The immunoreactive (IR) cells were observed under light microscope.

Cell counting and photomicrography. For each tissue, 5 sections were randomly chosen. All sections were examined and images were captured under Olympus BX61 microscope and photographed with an Olympus DP71 digital camera (Olympus, Tokyo, Japan). In each section, the number of IR cells in different areas selected at random was counted and positive cell density was calculated (positive cell density $=$ $=$ positive cell number/total cell number). 

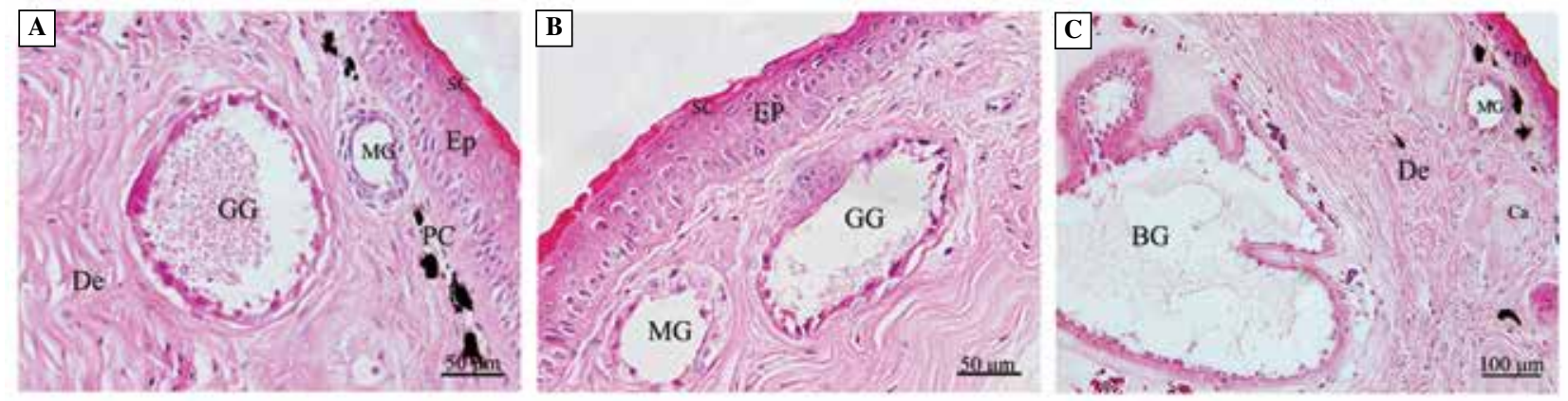

Figure 1. Histological structure of skin and parotoids of Bufo gargarizans, H\&E staining. A. Dorsal skin, scale bar $=50 \mu \mathrm{m}$. B. Ventral skin, scale bar $=50 \mu \mathrm{m}$. C. Parotoids. Scale bar $=100 \mu \mathrm{m}$. De - dermis; Ep — epidermis; SC - stratum corneum; GG — granular gland; MG — mucous gland; PC — pigment cell; BG — bottle-shaped gland; Ca — calcified cartilage zone

Statistical analysis. The data were expressed as means \pm standard deviations (means \pm SD) and the analysis of variances was performed by SPSS 13.0 software. Statistical significance was determined by One-Way ANOVA in the case of comparison of multiple groups. A $P<0.05$ was considered statistically significant.

\section{Results}

\section{The histological structure of the skin and parotoids of Bufo gargarizans}

Both the dorsal and ventral skin consists of two parts, epidermis and dermis (Figure 1A, B). Epidermis consists of stratum corneum, stratum granulosum, stratum spinosum and stratum germinativum. Dermis, located beneath the epidermis, is divided into dermis osteoporosis layer and dermal dense layer. Numerous glands including mucous and granular glands are distributed in dermis osteoporosis layer (Figure 1A, B). Mucous glands are small, with a hollow lumen or a few secretions. Granular glands are larger; the morphological structure varies during the different developmental stages: some glands are fully differentiated and filled with granular secretions in the lumen. On the contrary, other glands have fuzzy boundaries, and the lumen interspersed with cells. The dermal osteoporosis layer contains many melanin-containing pigment cells. Density layer located just beneath the osteoporosis layer is mainly composed of collagen fibers (Figure 1A, B).

The specialized parotoids are the biggest poison glands of Bufo gargarizans. Their secretion is an important source of toad venom. The structure of parotoids is similar to that of dorsal skin, composed of epidermis and the dermis (Figure 1C), but the epidermal layer is thinner than that of dorsal skin. Dermis osteoporosis layer contains granular, mucous and bottle-shaped glands as well as differentiated glands (Figure 1C). The simple tubular bottle-shaped glands make the main part of parotoids. The differentiated glands are made up of tall columnar cells which are full of secretions.

\section{The distribution of gastrointestinal hormone IR cells}

No positive labeling was seen in any of the negative control sections. The IR cells presented dark brown or yellow-brown, which was easy to be distinguished. Six types of GI hormone IR cells were detected in the skin and parotoids; they were 5-HT, GLU, GAS, SS, PP and NPY IR cells. The distribution density of these IR cells in the skin and parotoids were shown in Table 2.

\section{5-HT-IR cells}

In the dorsal skin, the 5-HT-IR cells distributed in all layers of epidermis and glands, and the distribution density among them had no significant difference $(P>0.05)$, but the immunoreactivity in granular glands was more intense than that in mucous glands (Figure 2A). In the ventral skin, the distribution density in stratum spinosum and stratum germinativum of epidermis was significantly higher than that in stratum corneum and stratum granulosum $(P<0.05)$, but no significant difference among the glands $(P>0.05)$. 5-HT existed in all cells of granular glands, not occurred in the duct opening of some mucous glands, and the immunoreactivity in granular glands was more intense than that in mucous glands (Figure 2B). In parotoids, the IR cells were abundantly distributed in mucous glands, granular glands, the inner layer of epidermis of bottle-shaped glands and differentiated 


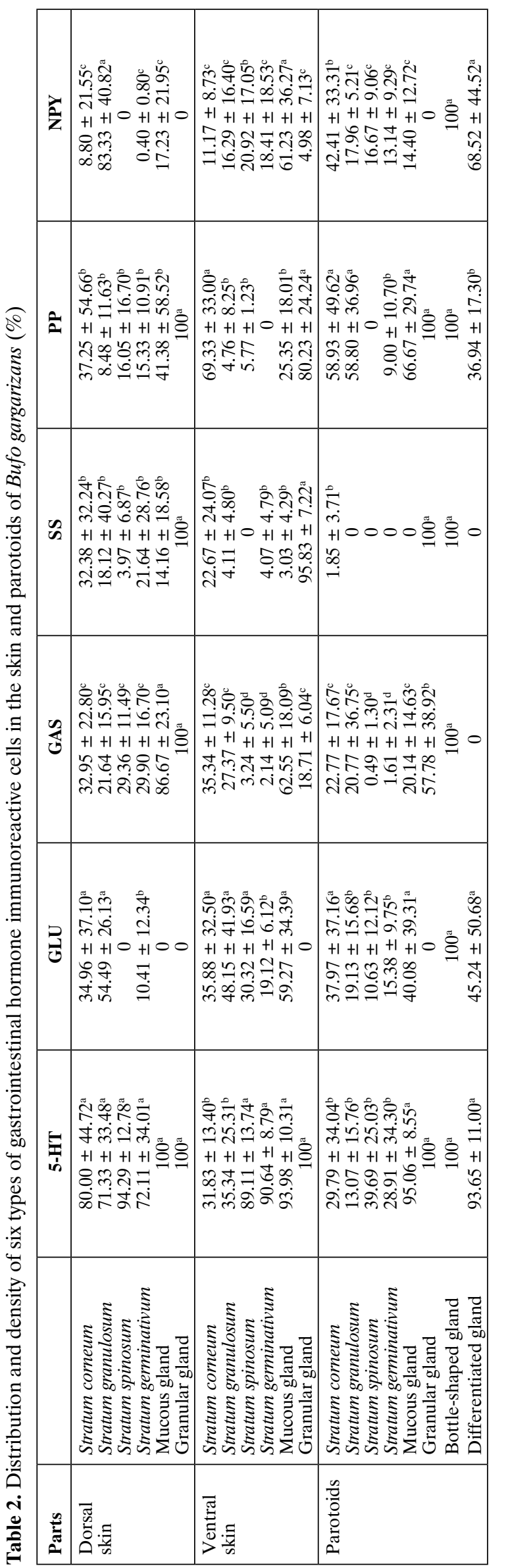

glands (Figure 2C). The distribution density in the glands was significantly higher than that in each layer of epidermis $(P<0.05)$, there was no significant difference among different layer of epidermis $(P>0.05)$.

\section{GLU-IR cells}

In the dorsal skin, the IR cells were distributed in the stratum corneum, stratum granulosum and stratum germinativum of epidermis, while not occurred in the stratum spinosum, granular glands and mucous glands (Figure 3A). The distribution density in stratum corneum and stratum granulosum were significantly higher than that in stratum germinativum $(P<0.05)$. In the ventral skin, GLU-IR cells were detected in all layers of epidermis and mucous glands, but were not found in granular glands (Figure 3B). The distribution density in stratum corneum, stratum granulosum, stratum spinosum and mucous glands were significantly higher than that in stratum germinativum $(P<0.05)$. In parotoids, the IR cells were distributed in all layers of epidermis (Figure 3C), the distribution density in stratum corneum was significantly higher than that in the other three layers of epidermis $(P<0.05)$. There was no significant difference among the mucous glands, bottle-shaped glands and differentiated glands $(P>0.05)$. No IR cells were detected in the granular glands.

\section{GAS-IR cells}

In the dorsal skin, the gastrin-IR cells were most predominant in mucous glands and granular glands (Figure 4A). The distribution density in the glands was significantly higher than that in each layer of epidermis $(P<0.05)$, and there was no significant difference among different layers of epidermis $(P>0.05)$. In the ventral skin, GAS-IR cells were distributed in both epidermis and glands. They were mainly present in stratum corneum and stratum granulosum, and rarely occurred in stratum spinosum and stratum germinativum (Figure 4B). The distribution density in mucous glands was significantly higher than that in granular glands and each layer of epidermis $(P<0.05)$. The immunoreactivity was most intense in granular glands. In parotoids, the IR cells were most predominant in stratum corneum and stratum granulosum, rarely occurred in stratum spinosum and stratum germinativum, and were absent in the differentiated glands (Figure 4C). The distribution densities in bottle-shaped glands and granular glands were significantly higher than that in mucous glands $(P<0.05)$.

\section{SS-IR cells}

In the dorsal skin, the IR cells were distributed in all layers of epidermis and glands, but mainly in granular 

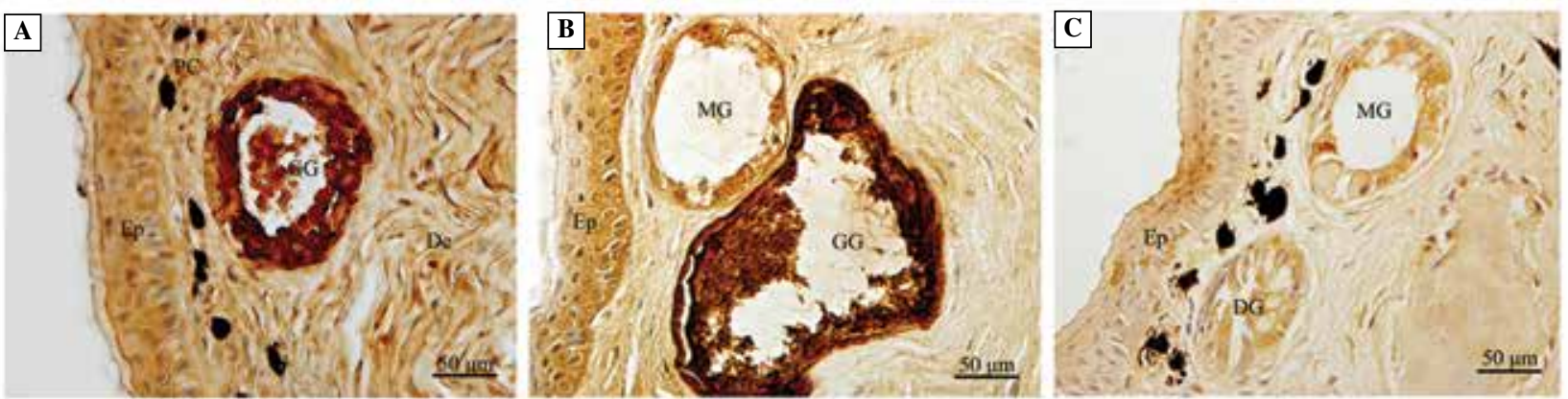

Figure 2. 5-HT-immunoreactive (IR) cells in skin and parotoids of Bufo gargarizans, IHC staining. A, B. The immunoreactivity of 5-HT-IR cells in the dorsal skin and ventral skin was most intense in GG. C. 5-HT-IR cells in the parotoids, cell density in the glands was higher than that in epidermis. Scale bar $=50 \mu \mathrm{m}$. Abbreviations as for Figure 1
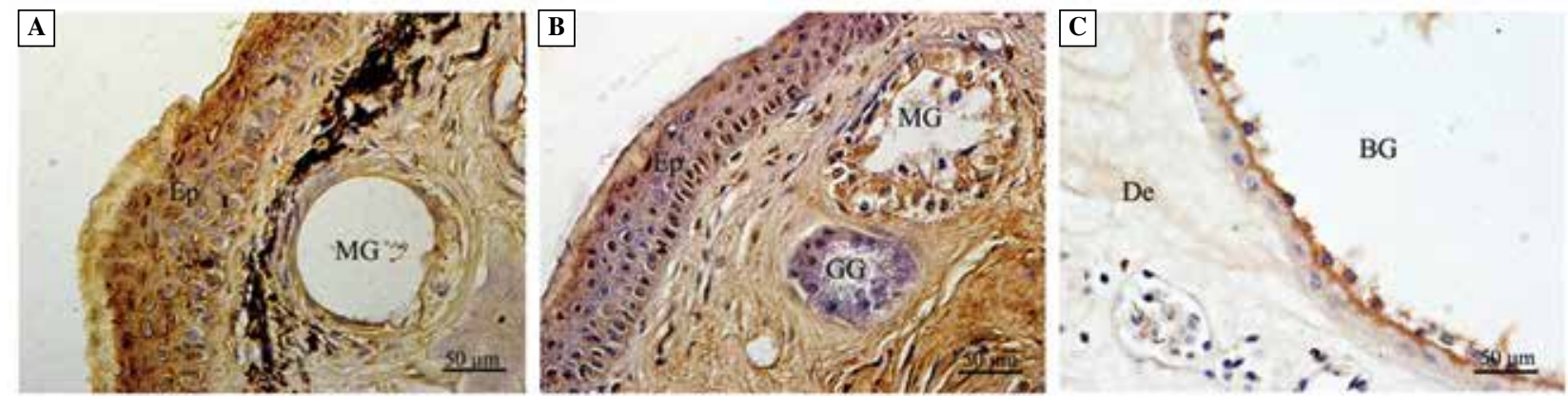

Figure 3. Glucagon-IR cells in skin and parotoids of Bufo gargarizans, IHC staining. A. GLU-IR cells in the Ep of dorsal skin, no IR cells were detected in the MG. B. GLU-IR cells in the ventral skin, no IR cells were detected in the GG. C. GLU-IR cells were abundant in the BG of the parotoids. Scale bar $=50 \mu \mathrm{m}$. Abbreviations as for Figure 1
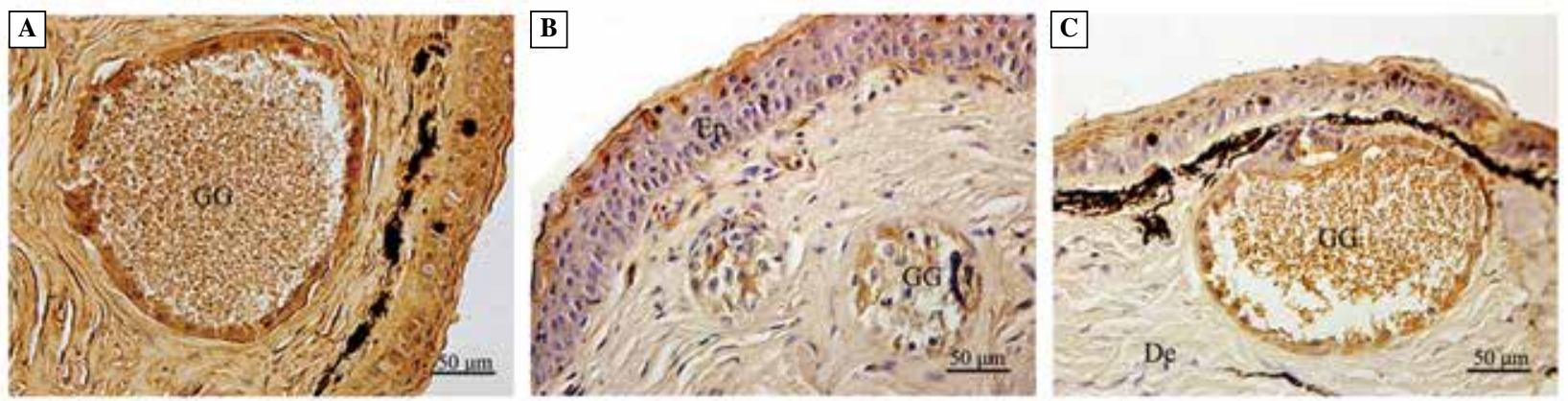

Figure 4. Gastrin-IR cells in skin and parotoids in skin and parotoids of Bufo gargarizans, IHC staining. A. GAS-IR cells were present in the GG of dorsal skin. B. GAS-IR cells in the ventral skin, the immunoreactivity was most intense in GG. C. GAS-IR cells in the GG of parotoids. Scale bar $=50 \mu \mathrm{m}$. Abbreviations as for Figure 1

glands (Figure 5A), and there were no significant differences among the other parts $(P>0.05)$. In the ventral skin, the distribution density of SS-IR cells in granular glands was higher than that in other parts, but these cells were absent in the duct opening of some granular glands; few SS-IR cells were also detected in other parts of ventral skin (Figure 5B). In parotoids, the IR cells were predominantly distributed in granular glands and the inner layer of epidermis of bottle-shaped glands, only occasionally detected in stratum corneum, not detected in the other parts of parotoids (Figure 5C).

\section{PP-IR cells}

In the dorsal skin, the pancreatic polypeptide-IR cells were distributed in all layers of epidermis (Figure 6A), 

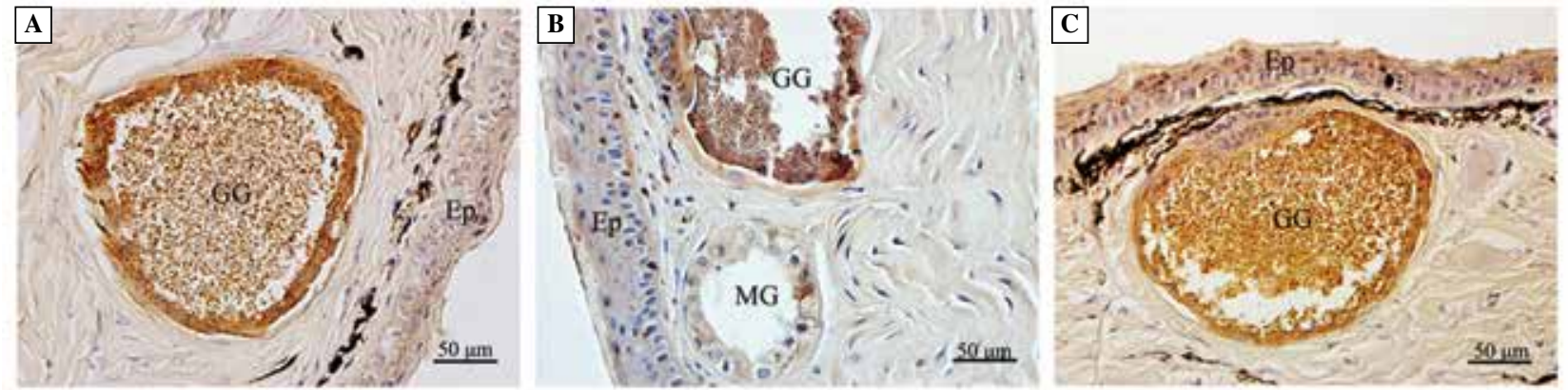

Figure 5. Somatostatin-IR cells in skin and parotoids of Bufo gargarizans, IHC staining. SS-IR cells were most predominant in the GG of the dorsal skin (A), ventral skin $(\mathbf{B})$ and parotoids $(\mathbf{C})$. Scale bar $=50 \mu \mathrm{m}$. Abbreviations as for Figure 1
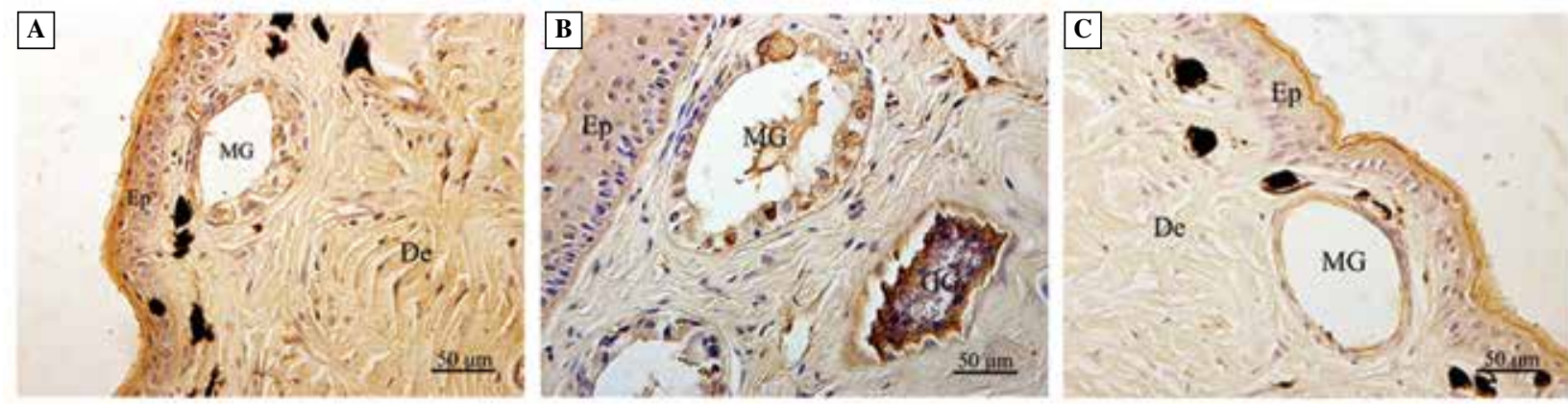

Figure 6. Pancreatic polypeptide-IR cells in skin and parotoids of Bufo gargarizans, IHC staining. A. PP-IR cells in the dorsal skin, the immunoreactivity was weak in Ep and MG. B. PP-IR cells in the ventral skin. The immunoreactivity was more intense in GG. C. PP-IR cells in the epidermis of parotoids, mainly present in stratum corneum and stratum granulosum. Scale bar $=50 \mu \mathrm{m}$. Abbreviations as for Figure 1

and there were no significant differences in the distribution density $(P>0.05)$. In the epidermis of ventral skin, PP-IR cells were most predominant in stratum corneum, and rarely occurred in stratum granulosum and stratum spinosum, and were not found in the stratum germinativum. The density of PP-IR cells in granular glands was significantly higher than that in mucous glands $(P<0.05)$, and the immunoreactivity was stronger than that in dorsal skin, while some of granular glands were not IR in the duct opening (Figure 6B). In the epidermis of parotoids, PP-IR cells were mainly distributed in stratum corneum and stratum granulosum, rarely occurred in stratum germinativum, and were not present in stratum spinosum (Figure 6C). PP-IR cells were distributed in all types of glands, but the distribution density in mucous glands, granular glands and bottle-shaped glands were significantly higher than that in differentiated glands $(P<0.05)$.

\section{NPY-IR cells}

In the epidermis of dorsal skin, NPY-IR cells were most predominant in stratum granulosum, rarely occurred in stratum corneum and stratum germinativum, and were not found in stratum spinosum. In addition, only some of the mucous glands were immunoreactive, and no immunoreactivity was detected in granular glands (Figure 7A). In the epidermis of ventral skin, the distribution density of NPY-IR cells in stratum spinosum was significantly higher than that in stratum corneum, stratum granulosum and stratum germinativum $(P<0.05)$. In addition, the distribution density of the NPY-IR cells in mucous glands was significantly higher compared to other parts $(P<0.05)$, but the immunoreactivity was weaker. The IR cells were only occasionally detected in the granular glands of ventral skin (Figure 7B). In parotoids, NPY-IR cells were predominantly distributed in stratum corneum; the distribution density in bottle-shaped glands and the differentiated glands was significantly higher than that in mucous glands $(P<0.05)$. No NPY-IR cells were detected in granular glands (Figure 7C).

\section{Discussion}

Serotonin (5-HT), glucagon, gastrin, somatostatin, pancreatic polypeptide and neuropeptide $\mathrm{Y}$ are 

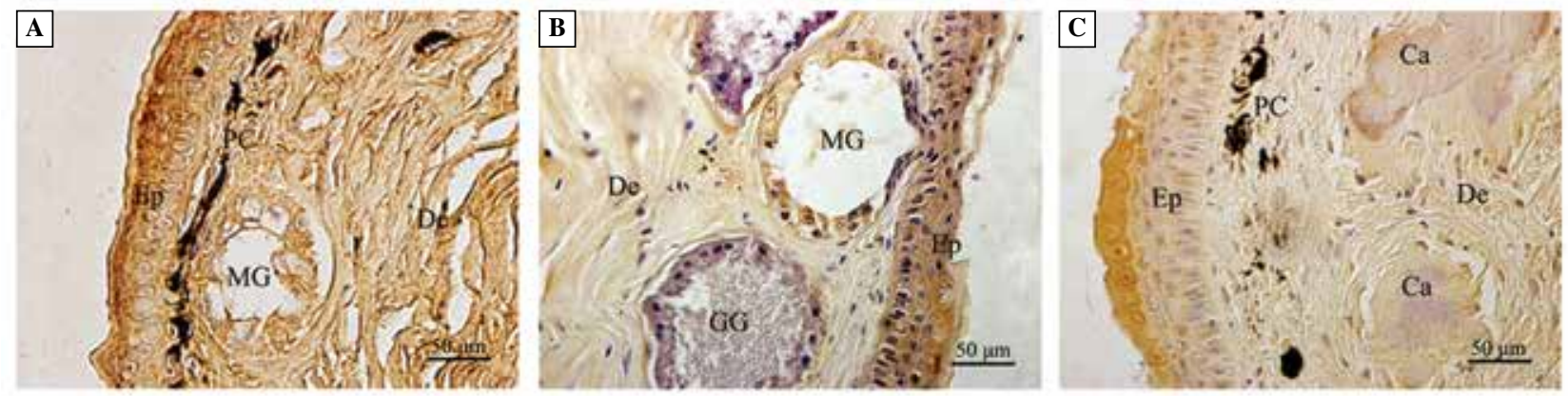

Figure 7. Neuropeptide Y-IR cells in skin and parotoids Bufo gargarizans, IHC staining. A. NPY-IR cells were most predominant in stratum granulosum of the dorsal skin. B. NPY-IR cells were most predominant in the MG of ventral skin. C. NPY-IR cells were predominant in stratum corneum of the parotoids. Scale bar $=50 \mu \mathrm{m}$. Abbreviations as for Figure 1

important types of GI hormones of vertebrates. We have previously detected by immunohistochemistry the presence of these GI hormones in the cells of the digestive system of several amphibians [11-13]. In this study, we found for the first time that these GI hormones IR cells also existed in the skin and parotoids of Bufo gargarizans.

It is remarkable that serotonin $(5-\mathrm{HT})$ was detected in the skin of various amphibian species [2-4]. 5-HT, a monoamine, could stimulate smooth muscle contraction and regulate ion selection and permeability of epithelial cells, processes which play a key role in water exchange of frog's skin [3, 14]. Some studies demonstrated that pigment cells of amphibians contain 5-HT receptor, and indicated that 5-HT had physiological effects on skin color changes [15]. In our present study, the positive reaction of 5-HT is the strongest among these six GI hormones, in particular, we found the higher immunoreactivity in the skin glands, what suggests that 5-HT may be also important in self-defense and regulation of the secretion of glands.

Until now there was only one study which identified and characterized of glucagon presence in amphibian (Rana rugulosa) skin [7]. Glucagon can stimulate glycogen decomposition, and increase the blood glucose levels. Our investigation indicated that GLU-IR cells existed both in the skin and parotoids, but were not present in granular glands; their function in the amphibian skin is yet to be further determined.

Gastrin can regulate the secretion of gastric acid and gastric fluid in the gastrointestinal tract and promote mucosal epithelial cell proliferation [16]. Greenwell and Low found that exogenous gastrin had an important effect on the active transport of sodium across the frog skin [17]. In this study, we found that GAS-IR cells existed in the skin and parotoids and that these cells were more prevalent in the glands, suggesting that gastrin in the amphibian skin could not only regulate the activity of epithelial cells, but it also may have an effect on the secretion of mucous glands.

Somatostatin is an inhibitory gut hormone which can inhibit activity of gastrin-secreting cells via a paracrine way, thus reducing the secretion of gastric acid [16]. In this study, we found SS-IR cells in the skin and parotoids of Bufo gargarizans, moreover, the distribution of SS-IR cells were similar to that of GAS-IR cells, suggesting that SS may regulate the activity of gastrin-containing cells in the skin similarly as in GI tract.

Pancreatic polypeptide (PP), peptide YY (PYY) and neuropeptide $\mathrm{Y}$ (NPY) are three members of pancreatic polypeptide family of structurally related peptides which are distributed in brain, peripheral nerves and the digestive tract playing important roles in the brain-gut axis. Using a sequence-specific radioimmunoassay and gel-chromatography, de Magistris et al. detected the bioactive peptides in the skin of 84 amphibian species and found that only $40 \%$ of the samples showed PP-like immunoreactivity, moreover, the content of PP-positive substance was very low [5]. In this study, we detected PP-IR cells in the skin and parotoids of Bufo gargarizans, but PP immunoreactivity was rather weak. NPY, a strong orexigenic peptide, is involved in the regulation of food intake and plays an important role in the regulation of neuroendocrine axis in adverse metabolic conditions [18]. In addition, NPY affects vascular smooth muscle contraction, blood pressure, and atherogenic processes [19]. We here reported for the first time the presence of NPY immunoreactivity in the amphibian skin. The epidermis of Bufo gargarizans contains rich capillary network, therefore, we inferred that NPY may also have the vasoactive effects in amphibian skin.

Gastrointestinal hormones are important in the regulation of a variety of different physiological processes. Some of the GI hormones or their analogs have been used for diagnosis and treatment of some 
diseases. For example, glucagon has been used to reduce pancreatic exocrine secretion, gastrin - in the treatment of atrophic gastritis [7], somatostatin in the treatment of psoriasis, rheumatoid arthritis and other diseases [20]. Our study not only provided a new theoretical basis for the medical use of Chan $\mathrm{Pi}$ and Chansu of Bufo gargarizans, but has also proposed its skin and parotoids as potential source for the extraction of the GI hormones.

\section{References}

1. Wei SS, Chi TT, Meng AY et al. Characteristics of hemolytic activity induced by skin secretions of the frog Kaloula pulchra hainana.J Venom Anim Toxins Incl Trop Dis. 2013;19:1-6.

2. Kramer B. Histochemical demonstration of 5-hydroxytryptamine in poison glands of amphibian skin. Histochemie. 1970;24:336-342.

3. Sengezer-Inceli M, Suren S, Murathanoglu O, Kaptan E. Immunohistochemical detection of serotonin in the skin of frogs (Rana ridibunda) kept at different temperatures. Biologia Bratislava. 2004;59:273-281.

4. Yoshie S, Iwanaga T, Fujita T. Coexistence of bombesin and 5-hydroxytryptamine in the cutaneous gland of the frog, Bombina orientalis. Cell Tissue Res. 1985;239:25-29.

5. de Magistris L, Annibale B, Delle Fave G et al. Peptides of the APUD system in amphibian skins. Peptides. 1985;6(Suppl 3): 203-208.

6. Liu XH, Wang YP, Cheng LH, Song YZ, Lai R. Isolation and cDNA cloning of cholecystokinin from the skin of Rana nigrovittata. Peptides. 2007;28:1540-1544.

7. Li Y, Li CC, Jin L, Dai GY, Zhang SZ. Distribution of five types of gastrointestinal hormone immunoreactive cells in the skin of Rana rugulosa. Chin J Anatomy. 2010;33:321-323.

8. Wang DL, Qi FH, Tang W et al. Chemical constituents and bioactivities of the skin of Bufo bufo gargarizans Cantor. Chem Biodivers. 2011:8:559-567.
9. Wang PS, Yeh JY, Yu CH, Wang SW. An evidence-based perspective of Bufo gargarizans (asiatic toad) for cancer patients. Evidence-based Anticancer Materia Medica. 2011; 389-407.

10. Qi F, Li A, Inagaki Y et al. Antitumor activity of extracts and compounds from the skin of the toad Bufo bufo gargarizans Cantor. Int Immunopharmacol. 2011;11:342-349.

11. Zhang SZ, Zhang S, Liu TT, Chen HH, Pan HZ. Distribution and morphology of endocrine cells in the digestive tract of Bufo gargarizans. Chin J Anat. 2008;31:519-521.

12. Pan HZ, Chen HH, Zhu LJ, Li Y, Li CC, Zhang SZ. Adaptive changes of gastro-entero-pancreatic system endocrine cells in the black-spotted pond frog Rana nigromaculata after fasting. Current Zool. 2009;55:301-308.

13. Huang $\mathrm{XG}, \mathrm{Wu} \mathrm{XB}$. Immunohistochemical localization of endocrine cells in the gastrointestinal tract of Mt. Wuyi Cascade-Frog (Amolops Wuyiensis). Acta Hydrobiol Sin. 2007;31:162-168.

14. Karan M, Timurkaan S, Aydin A. Serotonin immunoreactivity in the skin of the porcupine (Hystrix cristata). Veterinarski Arhiv. 2011;81:765-771.

15. Ali SA, Salim S, Sahni T, Peter J, Ali AS. 5-HT receptors as novel targets for optimizing pigmentary responses in dorsal skin melanophores of frog, Hoplobatrachus tigerinus. $\mathrm{Br} \mathrm{J}$ Pharmacol. 2012;165:1515-1525.

16. Friis-Hansen L. Lessons from the gastrin knockout mice. Regul Pept. 2007;39:5-22.

17. Greenwell JR, Low HS. Action of caerulein, gastrin 17, pentagastrin, and secretin on the active transport of sodium by the frog skin. J Membr Biol. 1981;61:91-96.

18. Pedrazzini T, Pralong F, Grouzmann E. Neuropeptide Y: the universal soldier. Cell Molec Life Sci. 2003;60:350-377.

19. McDermott BJ, Bell D. NPY and cardiac diseases. Curr Topics Med Chem. 2007;7:1692-1703.

20. Pinter E, Helyes Z, Nemeth J, Szolcsanyi J. Somatostatin as an anti-inflammatory neuropeptide: from physiological basis to drug development. Neuroimmune Biol. 2009;8: $119-134$. 